\title{
COMPARAÇÃO DOS FATORES DE RETARDAMENTO DO FOSFATO OBTIDOS COM DOIS MÉTODOS
}

\author{
Vanessa Almeida de Godoy ${ }^{1}$
}

Lázaro Valentin Zuquette ${ }^{2}$

Gian Franco Napa-García ${ }^{3}$

\begin{abstract}
RESUMO
Esta pesquisa teve como objetivo obter o fator de retardamento $(\mathrm{Rd})$ do fosfato $\left(\mathrm{PO}_{4}{ }^{3-}\right)$ por meio do método proposto por Freeze e Cherry (1979) e também por aquele proposto por van Genuchten e Parker (1984) e comparar os resultados, relacionando-os com as características do solo. Para isso, foram realizados ensaios em colunas indeformadas de solo arenoso por meio da percolação de três soluções de $\left(\mathrm{NH}_{4}\right)_{2} \mathrm{HPO}$ durante 20 volumes de poros. $\mathrm{O}$ retardamento do $\mathrm{PO}_{4}{ }_{4}^{3-}$ no solo estudado deve estar associado tanto à adsorção específica por óxidos de alumínio e ferro quanto às características físicas de dupla porosidade que podem reduzir a velocidade do $\mathrm{PO}_{4}{ }^{3-}$ quando o mesmo passar por poros menores e não conectados. Os valores de Rd obtidos com o método de van Genuchten e Parker (1984) são $18.5 \%$ maiores do que aqueles obtidos com o método de Freeze e Cherry (1979). No entanto, o método de Freeze e Cherry (1979) assume que a sorção no material obedece a uma isoterma linear, o que nem sempre representa o comportamento real do soluto. Por outro lado, o proposto por van Genuchten e Parker (1984) considera o balanço de massa no solo, podendo ser mais realista em termos do comportamento do soluto. Concluiu-se que a obtenção do Rd deve ser feita analisando-se tanto o modelo conceitual dos métodos propostos, bem como as características do solo estudado. Com isso é possível que se obtenham resultados mais confiáveis de Rd, o que resultara em uma melhor predição do transporte do $\mathrm{PO}_{4}{ }^{3-}$ no solo.
\end{abstract}

PALAVRAS-CHAVE: Fator de Retardamento. Fosfato. Dois Métodos.

\footnotetext{
${ }^{1}$ Doutoranda em Geotecnia, Universidade de São Paulo. valmeida@usp.br.

2 Prof. Livre-Docente, Departamento de Geotecnia, Universidade de São Paulo. lazarus1@sc.usp.br.

${ }^{3}$ Doutorando em Geotecnia, Universidade de São Paulo. gnapa@sc.usp.br.
} 


\title{
COMPARISON OF PHOSPHATE RETARDATION FACTORS OBTAINED BY TWO METHODS
}

\begin{abstract}
The first purpose of this paper is to obtain the retardation factor (Rd) of the phosphate $\left(\mathrm{PO}_{4}{ }^{3-}\right)$ using the method proposed by Freeze and Cherry (1979) and the method proposed by van Genuchten and Parker (1984). Then, the results were compared and related with the soil characteristics. For these purposes, laboratorial tests in undisturbed sandy soil columns were performed by percolation of three $\left(\mathrm{NH}_{4}\right)_{2} \mathrm{HPO}$ solutions during 20 pore volume. $\mathrm{PO}_{4}{ }^{3-}$ retardation in the studied soil can be associated with specific adsorption by aluminum and iron oxides as well as the physical characteristics, as dual porosity that can reduce the velocity of $\mathrm{PO}_{4}{ }^{3-}$ solution when it is moving through smaller and unconnected pores. The Rd values obtained with the method proposed by van Genuchten and Parker (1984) are 17\% higher than those obtained by the method proposed by Freeze and Cherry (1979). However, the method proposed by Freeze and Cherry (1979) considers that the sorption obeys a linear isotherm being that this is not always verified for the real behavior of the solute. On the other hand, the method proposed by van Genuchten and Parker (1984) considers the mass balance in the soil and may be more realistic. We conclude that the Rd estimation should be done by analyzing both the conceptual model of the proposed methods, as well as soil characteristics. Therefore, it is possible obtain more reliable values of Rd, which will allow a better prediction of the $\mathrm{PO}_{4}{ }^{3-}$ transport on the soil.
\end{abstract}

KEY-WORDS: Retardation factor. Phosphate. Two methods.

\section{COMPARACIÓN DE LOS FACTORES DE RETARDO DEL FOSFATO OBTENIDOS POR DOS MÉTODOS}

\section{RESUMEN}

El primer objetivo de este trabajo es obtener el factor de retardo (Rd) del fosfato $\left(\mathrm{PO}_{4}{ }^{3-}\right)$ utilizando el método propuesto por Freeze y Cherry (1979) y el método propuesto por van Genuchten y Parker (1984). Después, se compararon los resultados y los relacionaron con las características del suelo. Para esto, estudios laboratoriales fueron hechos en columnas no perturbadas de suelo arenoso por medio de la percolación de soluciones de $\left(\mathrm{NH}_{4}\right)_{2} \mathrm{HPO}$ durante 20 volúmenes de poros. El retardo del $\mathrm{PO}_{4}{ }^{3-}$ en el suelo estudiado puede estar relacionado con la adsorción específica por óxidos de aluminio y hierro, pero también con las características físicas como la dupla porosidad que puede reducir la velocidad de la solución de $\mathrm{PO}_{4}{ }^{3-}$ cuando este moviéndose por poros pequeños y no conectados. Los valúes de Rd obtenidos con él método propuesto por van Genuchten y Parker (1984) son $17 \%$ mayores que aquellos obtenidos con el método propuesto por Freeze y Cherry (1979). Sin embargo, el método propuesto por Freeze y Cherry (1979) adopta que la sorción obedece a una isoterma linear y eso no siempre representa el comportamiento real del soluto en el suelo. Por otro lado, el método propuesto por van Genuchten y Parker (1984) considera el balance de masa en el suelo y por esta razón puede ser más realista. Nosotros concluimos que la obtención del Rd debería ser realizada analizando tanto el modelo conceptual del método utilizado cuanto las características del suelo. Con eso, es posible obtener valores de Rd más confiables, lo que permitirá una mejor previsión del transporte de $\mathrm{PO}_{4}{ }^{3-}$ en el suelo.

PALABRAS-CLAVE: Factor de retardo. Fosfato. Dos métodos. 


\section{INTRODUÇÃO}

O uso de efluentes de esgotos domésticos na irrigação de terras agrícolas, técnica chamada de fertirrigação, é uma solução muito interessante e vantajosa em regiões onde existem solos pobres em matéria orgânica e em nutrientes, bem como em áreas onde há escassez de água para irrigação. Além disso, com a fertirrigação há possibilidade de redução da contaminação de corpos hídricos superficiais, redução do consumo de água potável e redução no uso de fertilizantes, reduzindo, também os custos da agricultura (TOZE, 2006; KIZILOGLU et al., 2007; RAMIREZ-FUENTES et al., 2002; HARUVY, 1997).

Segundo Toze (2006) e Kiziloglu et al. (2007), a principal desvantagem desta técnica é a possibilidade da inserção de altas concentrações de fosfato $\left(\mathrm{PO}_{4}{ }^{3-}\right)$ no solo, que pode ser transportado para camadas inferiores do solo e atingir os aqǘferos, contaminando-os e comprometendo a saúde e o abastecimento da população. $O$ fosfato pode ser absorvido pelos organismos vivos e causarem sérios problemas de saúde. Portanto, entender como o $\mathrm{PO}_{4}{ }^{3-}$ se movimenta no solo é uma tarefa importante tanto para o planejamento da irrigação com efluentes, quanto para a proteção das águas subterrâneas.

Contudo, devido à complexidade da previsão da movimentação de solutos no solo, a modelagem numérica muitas vezes se faz necessária. Um dos principais parâmetros de entrada dos modelos numéricos de transporte de solutos, o fator de retardamento $(\mathrm{Rd})$ pode ser obtido por meio de diversos métodos que, muitas vezes, são utilizados apenas com a consideração de sua simplicidade de aplicação, sem uma análise crítica do modelo conceitual, bem como das características do solo (GODOY, 2013). Assim sendo, esta pesquisa objetivou obter o $\mathrm{Rd}$ do $\mathrm{PO}_{4}{ }^{3-}$ por meio de dois métodos para que se comparassem os resultados, fazendo uma análise tanto dos modelos conceituais relacionados aos métodos, quanto das características do solo. 


\section{2 ÁREA DE ESTUDO}

A área de estudo (Figura 1) localiza-se no município de Cabrália Paulista que se encontra na região Centro-Oeste do Estado de São Paulo, com altitude média de $511 \mathrm{~m}$. De acordo com a classificação climática de Köppen, o clima da região de Cabrália Paulista é classificado como Cfa, ou seja, clima subtropical úmido com Verão quente. Os principais tipos de solos identificados em Cabrália Paulista são Latossolo, Gleissolo e Argissolo.

Figura 1: Localização da área de estudo

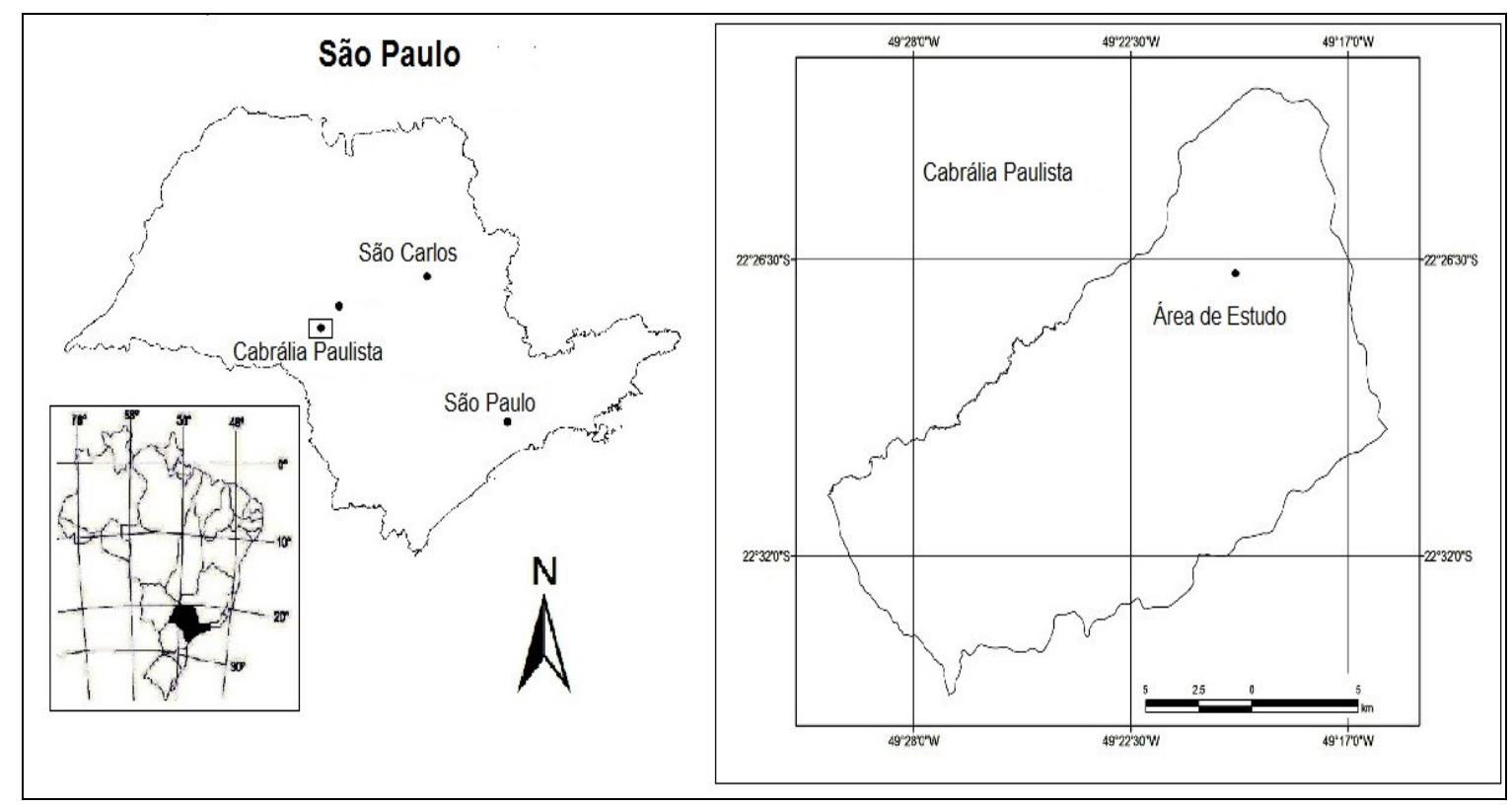

Cabrália Paulista situa-se sobre litologias encontradas na Bacia Sedimentar do Paraná, pertencentes ao Grupo Bauru que compreende um pacote sedimentar de idade Cretácea, aflorando em área de cerca de $117.000 \mathrm{~km}^{2}$ no Estado de São Paulo (PAULA E SILVA et al., 2003). A área de estudo compreende material inconsolidado residual de arenitos da Formação Adamantina. Hidrogeologicamente, Cabrália Paulista situa-se sobre o Sistema Aquifero Bauru (SAB), que ocupa aproximadamente a metade oeste do território do Estado de São Paulo, possuindo uma area aproximada de $96.880 \mathrm{~km}^{2}$ (DAEE et al., 2005). 


\section{METODOLOGIA}

Foram realizados ensaios de condutividade hidráulica in-situ de acordo com ABGE (1996). Em campo foram coletadas amostras deformadas e indeformadas à profundidade de $60 \mathrm{~cm}$. Caracterizações mineralógicas foram realizadas em laboratório por meio da difração de raios X (AZAROFF; BUERGUER, 1953) e análise de energia dispersiva (EDS). $O$ solo foi caracterizado fisicamente por meio da análise granulométrica conjunta (ABNT-NBR7181, 1984), determinação da massa específica dos sólidos (ABNT-NBR 6508,1984) e análise de porosimetria por intrusão de mercúrio (WASHBURN, 1921). A determinação do $\mathrm{pH}\left(\mathrm{H}_{2} \mathrm{O}\right.$ e $\left.\mathrm{KCl}\right)$, Eh e condutividade elétrica (EC) (EMBRAPA, 1997), delta pH (MEKARU; UEHARA, 1972), ponto de carga zero (PCZ) (KENG; UEHARA, 1974), teor de matéria orgânica (ABNTNBR 13600, 1996) e Capacidade de troca (CEC)(PEJON, 1992) foram utilizadas para a caracterização físico-química do solo.

Em seguida iniciou-se o ensaio de coluna para determinação dos índices físicos do solo (NOGUEIRA, 2005) da velocidade linear média (v), velocidade de Darcy (q) e condutividade hidráulica (k). Depois, em cada uma das três colunas percolou-se uma solução de $\left(\mathrm{NH}_{4}\right)_{3} \mathrm{PO}_{4}{ }^{3-}(35,65$ e $95 \mathrm{ppm})$ até um total igual a 20 volumes de poros. Durante todo o ensaio de coluna, as amostras efluentes tiveram seu $\mathrm{pH}$, condutividade elétrica e temperatura monitorados.

Ao término do ensaio de percolação, as colunas foram lavadas com água destilada durante três volumes de poros. As amostras foram coletadas em cada volume de poros, armazenadas e refrigeradas. Para a determinação das concentrações $\mathrm{PO}_{4}{ }^{3-}$ foi necessário alcalinizar as soluções com pH variando de 13 a 14 (HAVLIN, et al., 2005). Depois de alcalinizadas, inseriu-se o reagente PhosVer $3 \mathrm{e}$ as determinações foram feitas com o espectrofotômetro HACH modelo 2010.Com isso, construíram-se as curvas de chegada de concentração relativa $\left(C / C_{0}\right)$ versus volume de poros.

A partir dessas curvas determinaram-se os $\mathrm{Rd}$ do fosfato por meio de dois métodos. O primeiro foi proposto por Freeze e Cherry (1979). Seguindo este método, 
o Rd é igual ao $\mathrm{Vp}$ quando a $\mathrm{C} / \mathrm{C}_{0}$ é 0,5 . Outro método utilizado foi proposto por van Genuchten e Parker (1984), onde o Rd é igual a área acima da curva de chegada assumindo que a mesma segue o modelo analítico proposto por Lapidus e Amundson (1952) e, depois, reescrito por Ogata (1958) e Bear (1972).

\section{RESULTADOS}

\subsection{CARACTERIZAÇÃO DO SOLO}

A Figura 2 mostra as curvas granulométricas do solo estudado, com e sem o uso de defloculante. Pode-se perceber que, como esperado, a análise com defloculante apresentou maior porcentagem de argila $(16,1 \%)$ do que a análise sem o uso de defloculante (10,5\%). Além disso, percebeu-se que o solo é predominantemente arenoso para os dois ensaios realizados (79,3 e 79,4\% de areia com e sem defloculante, respectivamente).

Figura 2: Curvas granulométricas do solo estudado

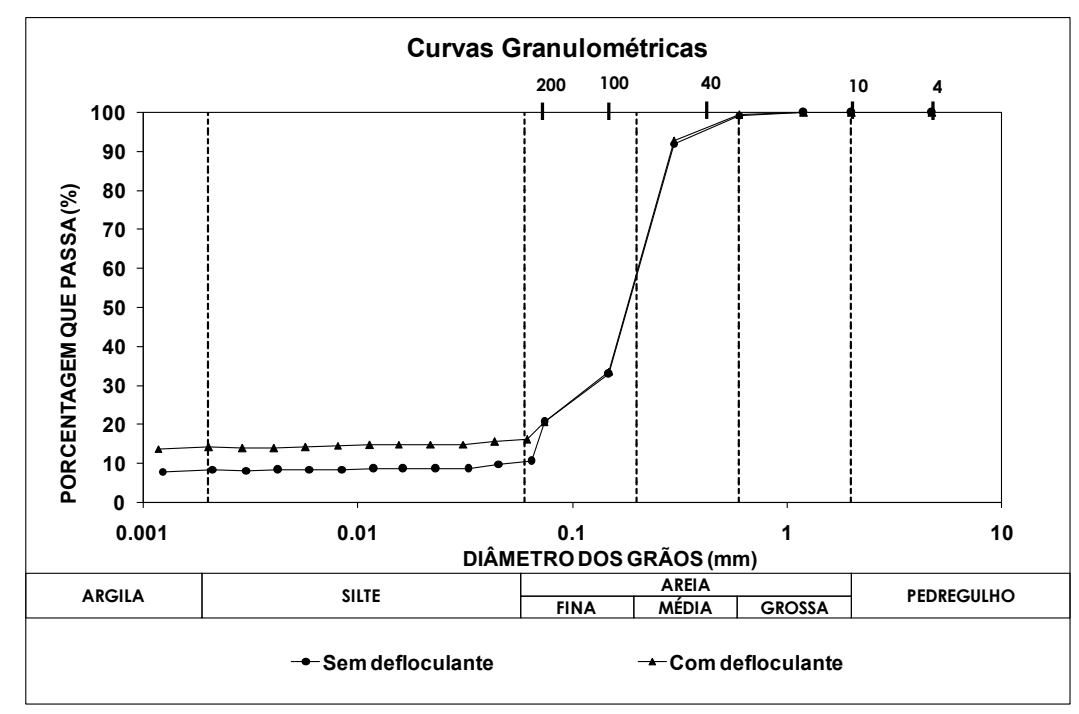


A mineralogia do solo é composta por quartzo, caulinita e gibsita, mineralogia típica para solos desta região. A análise de porosimetria por intrusão de mercúrio constatou a presença de dupla porosidade (Figura 2) com cerca de $5 \%$ de macroporos. Este solo possui teor de matéria orgânica de $1,18 \%$ e a massa específica dos sólidos mostra-se adequada para um solo arenoso $\left(26,4 \mathrm{kN} / \mathrm{m}^{3}\right)$.

Figura 3: Frequência versus diâmetro dos poros

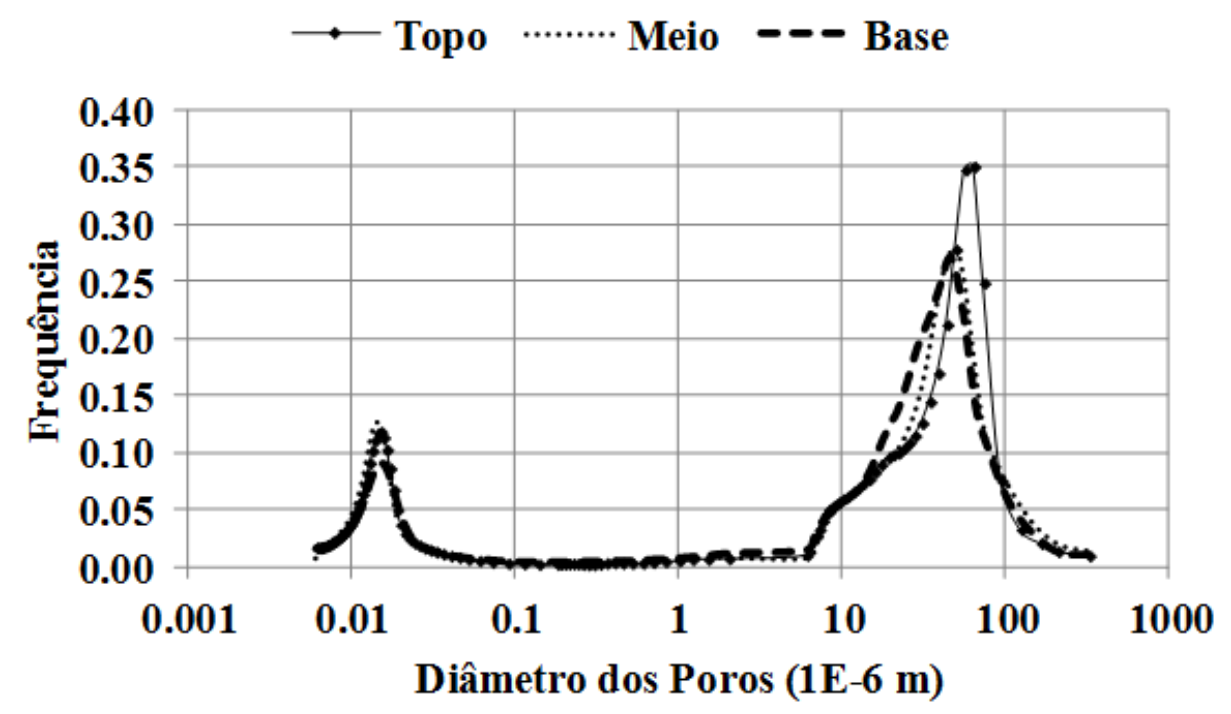

A condutividade elétrica indica a existência de baixas concentrações de sais dissolvidos $(55,70 \mathrm{mS} / \mathrm{m})$. De acordo com o valor do $\mathrm{pH}$ em $\mathrm{H}_{2} \mathrm{O}$ e em $\mathrm{KCl}(5,23 \mathrm{e}$ 4,20 respectivamente), o solo é ácido o que característico dessa região. $\mathrm{O}$ valor de CTC obtido $(0,152 \mathrm{cmol} / \mathrm{kg})$ indica que o material possui baixa capacidade de retenção de cátions por adsorção eletrostática. Com o $\Delta \mathrm{pH}$ negativo $(-1,03)$ e com o PCZ $(4,18)$ menor do que o pH do solo, verifica-se que há predomínio de cargas negativas no solo, o que pode prejudicar a adsorção de ânions como o $\mathrm{PO}_{4}{ }^{3-}$. A EDS indicou presença de $62,22 \%$ de oxigênio, $10,9 \%$ de alumínio e $2,26 \%$ ferro, que são importantes na adsorção específica de fosfato (HAVLIN, et al., 2005). 


\subsection{ENSAIO DE COLUNA}

Os valores de condutividade hidráulica são compatíveis com solos arenosos (com ordem de grandeza de 10E-5 m/s), e os valores da velocidade de Darcy e da linear média foram da ordem de 10-4 m/s. Estes dois valores foram similares entre si e este resultado é reflexo da macroporosidade do solo.

Durante os ensaios de coluna, foram monitorados o $\mathrm{pH}$, a temperatura e a condutividade elétrica. Os valores de $\mathrm{pH}$ variaram entre 6,8 e 7,7 e a temperatura variou de 22,5 a $23{ }^{\circ} \mathrm{C}$. Notou-se aumenta da condutividade elétrica com o passar do volume de poros. A Figura 4 mostra as três curvas de chegada do fosfato obtidas com os ensaios de coluna.

Figura 4: Curvas de chegada do fosfato

Curva de Chegada de $\mathrm{PO}_{4}{ }^{3-}$

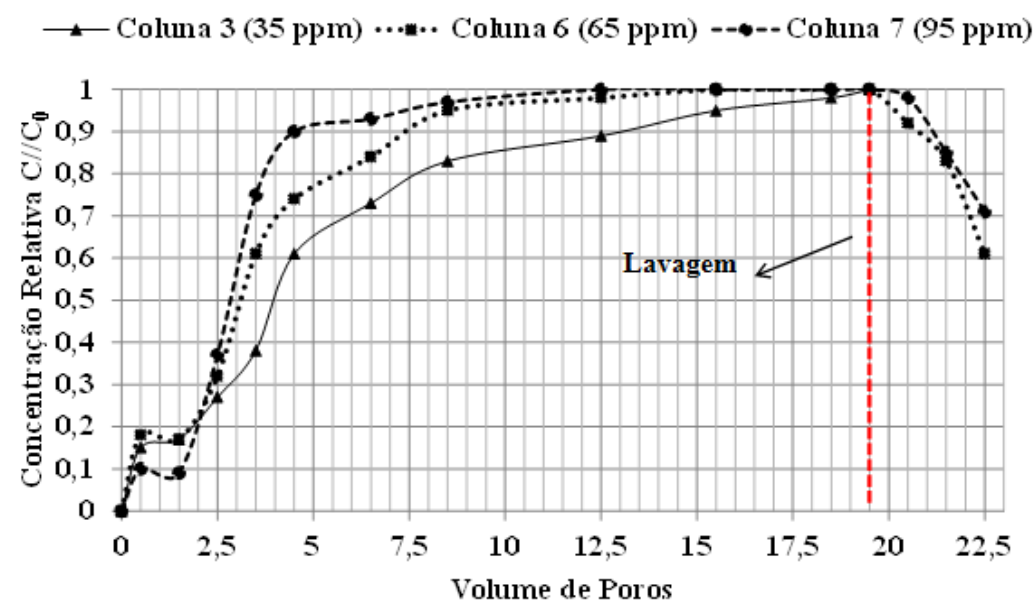

Observando a curva de chegada (Figura 4) é possível notar que houve significativa retenção do fosfato. Esta retenção pode ocorrer devido à adsorção específica causada pela presença de alumínio e ferro no solo. No entanto, a análise da Figura 4 também permite que se note a rápida movimentação do íon, visto que no primeiro volume de poros percolado já é constata sua presença na solução efluente. 
Assim, acredita-se que a presença de dupla porosidade pode estar reduzindo a mobilidade do fosfato em alguns locais na coluna de solo (macroporos e poros não conectados) e aumentando a mobilidade em outros locais, por exemplo, quando existem caminhos preferenciais, como macroporos interligados.

\subsection{OBTENÇÃO DO FATOR DE RETARDAMENTO}

As Figuras $5 a, 5 b$ e $5 c$ mostram a obtenção dos valores Rd e a Figura 6 mostra a comparação dos valores de Rd obtidos com os dois métodos utilizados nesta pesquisa.

Figura 5a: Obtenção Rd para $C_{0}$ de 35 ppm de fosfato

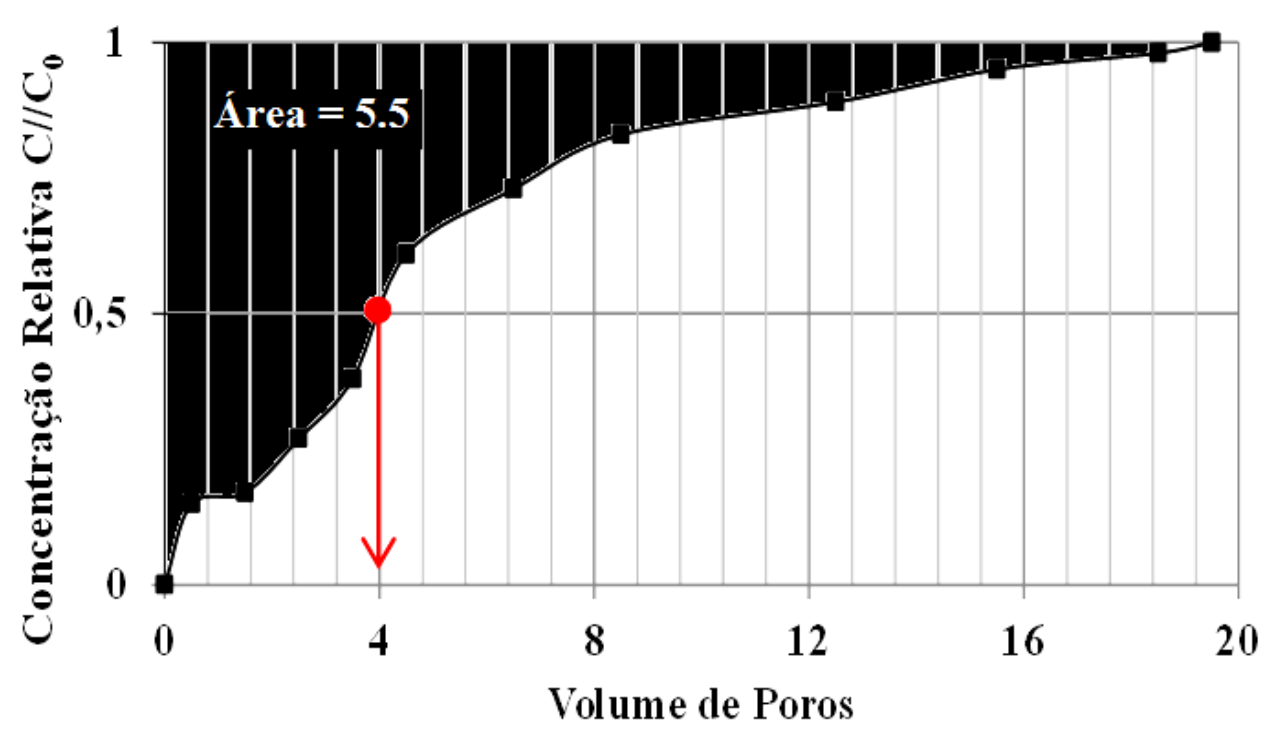


Figura 5b: Obtenção do Rd para $C_{0}$ de 65 ppm de fosfato

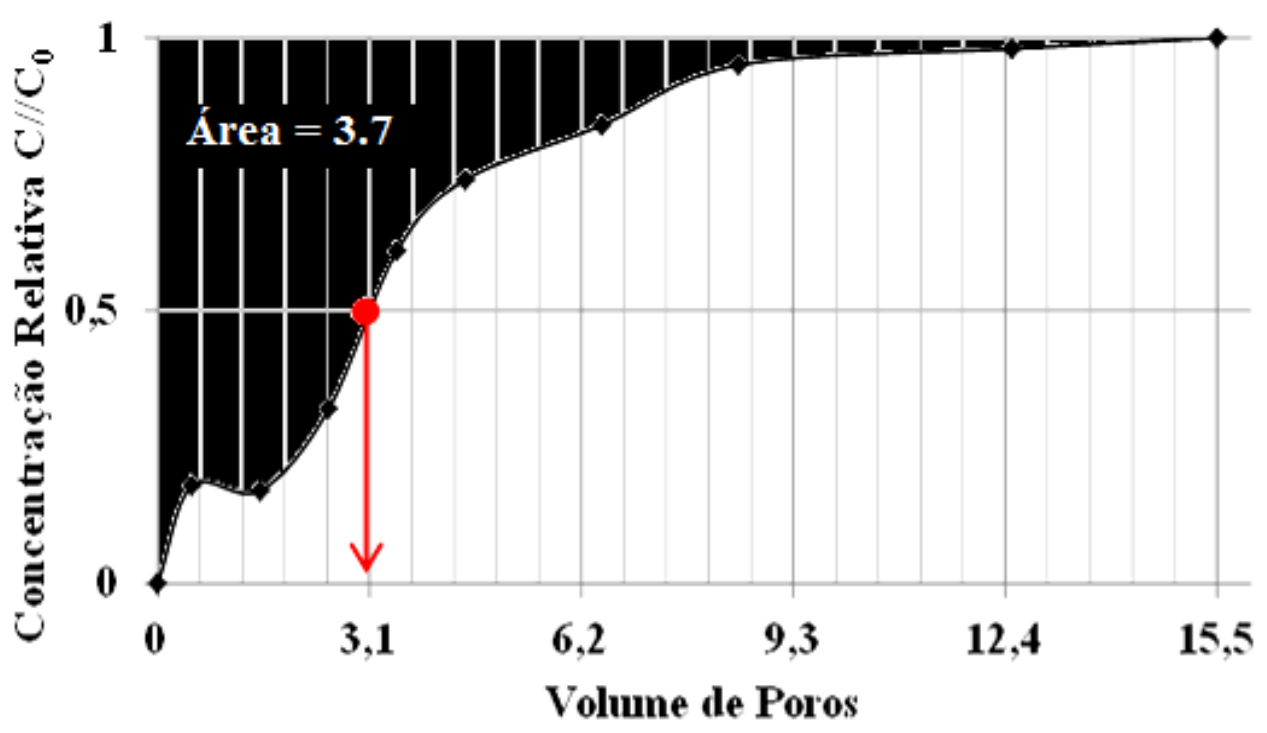

Figura 5c: Obtenção do Rd para $C_{0}$ de 95 ppm de fosfato

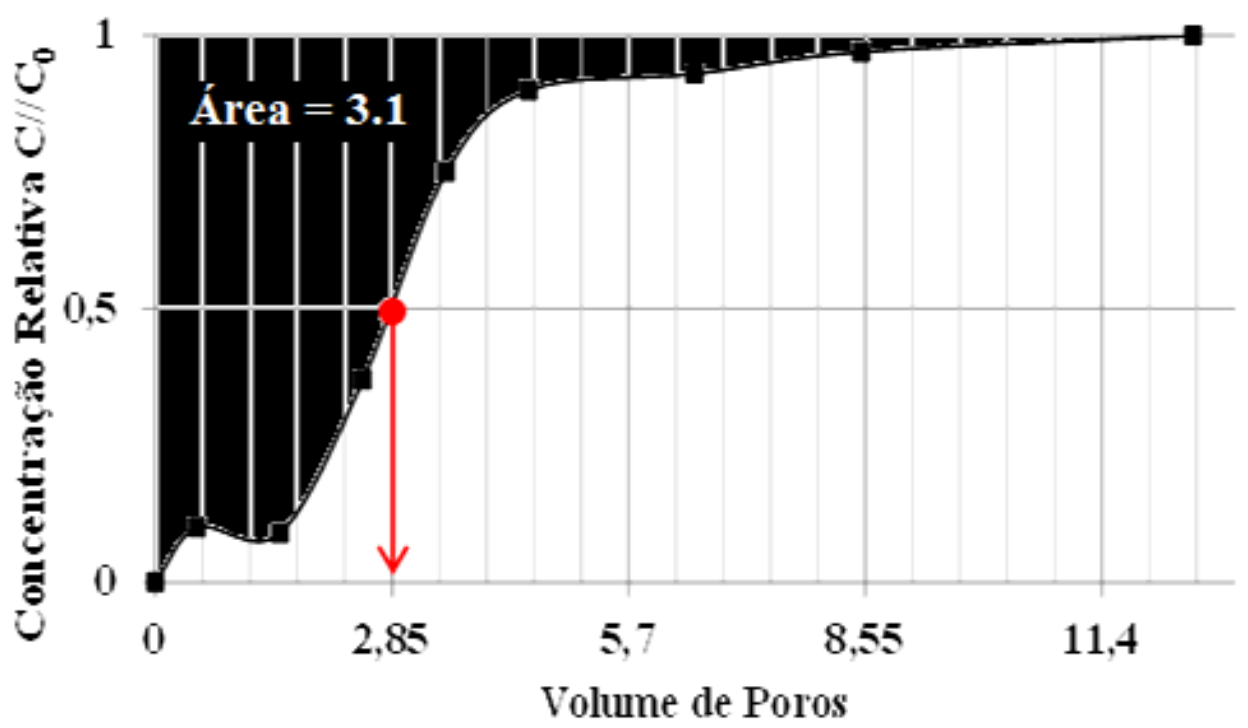


Figura 6: Comparação dos valores de Rd do fosfato obtidos pelos dois métodos utilizados

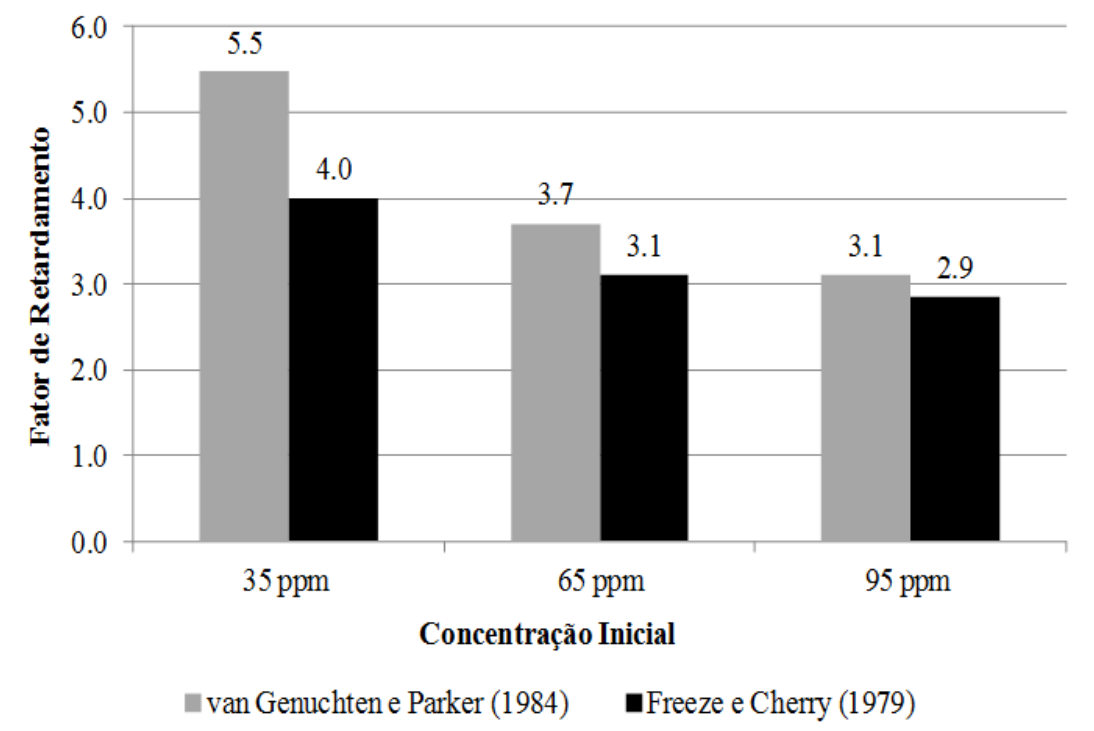

Percebe-se que para os métodos utilizados, quanto maior a concentração inicial, menor será o Rd. O que pode ser justificado pelo fato de que, para um mesmo solo, quanto maior a concentração do contaminante, maior será a quantidade de soluto que não será retido, uma vez que os sítios de sorção serão os mesmo e que eles serão ocupados por uma quantidade fixa de soluto. Desta forma, um acréscimo na quantidade de soluto fará com que mais soluto fique livre na solução do solo e seja, então, constatada na solução efluente da coluna de solo.

Os valores dos fatores de retardamentos obtidos com o método proposto por Freeze e Cherry (1979) são em média 18,5\% menores do que aqueles obtidos com o método proposto por van Genuchten e Parker (1984). No entanto, observa-se que as diferenças diminuem com o aumento da concentração inicial da solução contaminante (Figura 6). Assim, para o solo estudado, quando existirem altas concentrações de contaminantes, é possível que os dois métodos utilizados apresentem os mesmos valores de $\mathrm{Rd}$.

O método de Freeze e Cherry (1979) deve ser usado apenas quando se admite que a adsorção é rápida e reversível e que obedece a uma isoterma linear (FREEZE; CHERRY, 1979; SHACKELFORD, 1994). Contudo, este comportamento nem sempre representa o comportamento real do soluto, o que deve ser levado em 
consideração ao se utilizar os valores de $\mathrm{Rd}$ obtidos com este método. Ao se calcular a área acima da curva de chegada com o método de van Genuchten e Parker (1984), é considerado o balanço de massa no solo, podendo ser este um método que resulta em valores mais realistas em termos do comportamento do $\mathrm{PO}_{4}{ }^{3-}$ para este solo. De qualquer maneira, a análise das características do solo, bem como dos modelos conceituais dos métodos, pode auxiliar na escolha do mais adequado e com isso aumenta-se a confiabilidade do valor do $\mathrm{Rd}$ e, consequentemente, da modelagem numérica que for baseada neste dado.

\section{CONCLUSÕES}

Esta pesquisa realizou uma análise comparativa dos valores do fator de retardamento do fosfato obtidos com dois métodos, com o objetivo de analisar os modelos conceituais de cada método além de relacionar com as características do solo.

Ensaios de caracterização foram fundamentais para que se interpretassem resultados de retardamento obtidos. A presença de dupla porosidade e de macroporosidade influenciaram fortemente a determinação dos valores dos parâmetros de fluxo e do Rd. Além disso, a análise das características químicas, físico-químicas e mineralógicas auxiliaram no entendimento do comportamento de sorção do solo.

Verificou-se a presença tanto de advecção quanto de adsorção específica por óxidos e hidróxidos de alumínio e de ferro. O ferro, apesar de não ter sido constatado na DRX, está presente em forma não cristalina neste solo, como pôde ser verificado com as altas quantidades na análise de EDS.

A análise dos modelos conceituais dos métodos utilizados permitiu que se percebesse quando um método pode ser mais indicado do que outro. Assim pode haver uma análise mais cuidadosa dos valores obtidos, relacionando-os com as características físicas, químicas, físico-químicas e mineralógicas do solo. 
Notou-se que com o aumento das concentrações iniciais das soluções contaminantes, a diferença dos valores de Rd entre os dois métodos tendeu a diminuir. Assim, para este solo, em situações em que se trabalha com altas concentrações, é possível que os dois métodos sejam igualmente indicados.

Os resultados sugerem que $\mathrm{o}$ uso de parâmetros de transporte em modelagem do transporte de contaminantes deve ser feito com cautela. É necessário que se observe tanto as características do solo (mineralógicas, físicas, químicas, físico-químicas) quanto o modelo conceitual e as limitações dos métodos usados na determinação desses parâmetros.

\section{AGRADECIMENTOS}

Agradecemos aos professores e funcionários da Escola Técnica Estadual "Astor de Mattos Carvalho", à coordenação de Aperfeiçoamento de Pessoal de Nível Superior e à Escola de Engenharia de São Carlos da Universidade de São Paulo, por permitirem o desenvolvimento deste trabalho.

\section{REFERÊNCIAS}

ABGE. Ensaios de permeabilidade em solos - orientações para sua execução no campo. Boletim n 4. São Paulo, In: OLIVEIRA, Antônio Manuel Santos., CORRÊA FILHO, Diogo. (Coord.), 1996.

ABNT. NBR 13600-1996: Solo - Determinação do teor de matéria orgânica por queima a $440{ }^{\circ} \mathrm{C}$. Rio de Janeiro, 1996. 2p.

ABNT. NBR 6508-1984a: Grãos de solos que passam na peneira de 4,8 mm: determinação da massa específica: método de ensaio. Rio de Janeiro, $7 \mathrm{p}$.

ABNT. NBR 7181-1984c: Solo - análise granulométrica. Rio de Janeiro, 13 p.

AZAROFF, Leonid V; MARTIN, Julian Buerger. The powder method in X-ray crystallography, 1953. $342 p$.

BEAR, Jacob. Dynamics of fluids in porous media. American Elsevier, Nova York, 1972. 242p. 
DAEE et al. Mapa de água subterrânea do Estado de São Paulo Escala 1:1.000.000 - Nota Explicativa. São Paulo, 3v. 119p., 2005.

EMBRAPA. Centro Nacional de Pesquisa de Solos. Manual de métodos de análise de solo. Rio de Janeiro, 1997. 212p.

FREEZE, Allan. e CHERRY, John. A. Groundwater, Prentice-Hall, Englewood Cliffs, Nova Jersey, 1979. $604 \mathrm{p}$.

GODOY, Vanessa Almeida. Estudo dos mecanismos de transporte de sódio, fosfato e amônio em colunas indeformadas de material inconsolidado residual de arenitos da Formação Adamantina. 255p. Dissertação (Mestrado), Escola de Engenharia de São Carlos, Universidade de São Paulo, 2013.

HARUVY, Nava. Agricultural reuse of wastewater: nation-wide cost-benefit analysis. Agriculture, Ecosystems \& amp; Environment, v.66, n.2, p.113-119, 1997.

HAVLIN, John. et al. Soil fertility and fertilizers: An introduction to nutrient management. 7ed. Upper Saddle River, NJ: Pearson Prentice Hall, 2005. 515p.

KENG, John Ching-Win; Uehara, Goro. Chemistry, mineralogy and taxonomy of Oxisols and Ultisols. Proceedings Soil and Crop Science Society, v. 33, p. 119-126, 1974.

KIZILOGLU, Fatih. et al. Effects of wastewater irrigation on soil and cabbage-plant (brassica olerecea var. capitate cv. yalova-1) chemical properties. Journal of Plant Nutrition and Soil Science, v.170, n.1, p.166-172, 2007.

LAPIDUS, Leon; AMUNDSON, Neal. Mathematics of adsorption in beds. VI. The effect of longitudinal diffusion in ion exchange and chromatographic columns. The Journal of Physical Chemistry, v. 56, $n$. 8, p. 984-988, 1952.

MEKARU, Toshio; UEHARA, Goro. Anion adsorption in ferruginous tropical soils. Soil Science Society of America Journal, v. 36, n. 2, p. 296-300, 1972.

NOGUEIRA, João Batista. Mecânica dos Solos: ensaios de laboratório. São Carlos, SP: EESCUSP, 2005, 80p.

OGATA, Akio. Dispersion in porous media. 1958. 121 p. (Tese de Doutorado). Northwestern University, 1958.

PAULA E SILVA, Flávio; CHENG, Hung Kiang; CAETANO-CHANG, Mari Rita. Perfis de referência do Grupo Bauru (K) no Estado de São Paulo. Geociências, v. 22, n. especial, p. 21-32, 2003.

PEJON, Osni José. Mapeamento geotécnico regional da folha de Piracicaba-SP (escala 1.100.000) estudo de aspectos metodológicos, de caracterização e de apresentação de atributos. 1992. 2 vols., 224 p. Tese (Doutorado em Geotecnia) - Escola de Engenharia de São Carlos, Universidade de São Paulo, São Carlos, SP, 1992.

RAMIREZ-FUENTES, Edward. et al. Characteristics, and carbon and nitrogen dynamics in soil irrigated with wastewater for different lengths of time. Bioresource Technology, v.85, n.2, p.179-187, 2002.

TOZE, Simon. Reuse of effluent water: benefits and risks. Agricultural Water Management, v.80, n.13, p.147-159, 2006.

VAN GENUCHTEN, Martinus Theodore; PARKER, Joseph C. Boundary conditions for displacement 
Volume 10, Número 12, 2014

Saúde, Saneamento

e Meio Ambiente

experiments through short laboratory soil columns. Soil Science Society of America Journal, v. 48, n. 4, p. 703-708, 1984.

WASHBURN, Eduard Wight. The dynamics of capillary flow. Physical Review, v.17,p. 273-283, 1921. 